\title{
FACTORES DE RIESGO DE ANEMIA FERROPÉNICA EN NIÑOS MENORES DE UN AÑO
}

\section{RISKFACTORS FORIRON DEFICIENCYANEMIAIN CHILDRENUNDERONE YEAR OF AGE}

\author{
César Ramón Góngora-Ávila', Roberto Alejandro Mejias-Arencibia'1 , Lisandra Vázquez- \\ Carvajal², José Carlos Álvarez Hernández ${ }^{3}$, Annalie Elizabeth Frías Pérez ${ }^{4}$
}

\begin{abstract}
RESUMEN
Objetivo. Identificar los factores de riesgo de anemia ferropénica en niños menores de un año pertenecientes al Policlínico Docente "7 de Noviembre" del municipio Majibacoa durante el año 2020. Materiales y Métodos. Estudio observacional, descriptivo, de corte transversal en lactantes menores de un año con anemia ferropénica pertenecientes al Policlínico Docente "7 de Noviembre" del municipio Majibacoa durante el año 2020. El universo estuvo constituido por 42 niños menores de un año con anemia ferropénica; se trabajó con la totalidad de estos. Se analizaron las variables: edad, sexo, intensidad de la anemia, factores de riesgo biológicos y factores de riesgos sociales y culturales. Resultados. El 61,9 \% eran del sexo femenino; por su parte la edad más representada fue la menor de 6 meses en ambos sexos, aunque más observada en las féminas (33,3\%); el 61,9\% tenían anemia ligera. El 69,0\% de las madres con anemia gestacional sus hijos presentaron anemia ferropénica; por otra parte, el 47,6\% de los niños menores de un año tenían como antecedente el abandono de la lactancia materna exclusiva. Conclusiones. El antecedente de anemia durante la gestación, el bajo peso al nacer, la ablactación incorrecta y el abandono de la lactancia materna exclusiva constituyen los principales factores de riesgo en el origen de la anemia ferropénica en niños menores de un año.
\end{abstract}

Palabras claves: Lactantes; Anemia ferropénica; Déficit de hierro; Déficit nutricional. (Fuente: DeCS BIREME).

\begin{abstract}
Objective: To identify risk factors for iron deficiency anemia in children under one year of age belonging to the Teaching Polyclinic "November 7" of the Majibacoa municipality during the year 2020. Materials and Methods. Observational, descriptive, cross-sectional study in infants under one year of age with iron deficiency anemia belonging to the Teaching Polyclinic "7 de Noviembre" of the Majibacoa municipality during 2020. The universe consisted of 42 children under one year of age with iron deficiency anemia; all of these were worked on. The variables were analyzed: age, sex, intensity of anemia, biological risk factors, and social and cultural risk factors. Results. $61,9 \%$ were female; For its part, the age most represented was less than 6 months in both sexes, although it was more observed in females $(33,3 \%) ; 61,9 \%$ had mild anemia. $69,0 \%$ of mothers with gestational anemia, their children presented iron deficiency anemia; On the other hand, $47,6 \%$ of children under one year of age had a history of abandoning exclusive breastfeeding. Conclusions. The antecedent of anemia during pregnancy, low birth weight, incorrect ablactation and abandonment of exclusive breastfeeding are the main risk factors at the origin of iron deficiency anemia in children under one year of age.
\end{abstract}

Keywords: Infants; Iron deficiency anemia; Iron deficiency; Nutritional deficit. (Source: MeSH NLM).

\section{INTRODUCCIÓN}

La anemia es un problema generalizado que tiene consecuencias de gran alcance para la salud humana, así como en el desarrollo social y económico de los países. En la actualidad se relaciona estrechamente con la desnutrición y las enfermedades, esta suele utilizarse como indicador para estimar la calidad de los programas socio-sanitarios de las naciones ${ }^{1}$.

En el grupo de anemias carenciales la ferropénica o por déficit de hierro es la más frecuente, esta tiene como causa principal el déficit de hierro, con disminución en la síntesis de la hemoglobina en el eritroblasto².

\footnotetext{
Universidad de Ciencias Médicas de Las Tunas, Facultad de Ciencias Médicas “Dr. Zoilo Enrique Marinello Vidaurreta”. Las Tunas, Cuba.

Universidad de Ciencias Médicas de Ciego de Ávila. Facultad de Ciencias Médicas de Morón. Ciego de Ávila. Cuba.

Universidad de Ciencias Médicas de Ciego de Ávila. Policlínico Docente Universitario Sur. Morón. Ciego de Ávila. Cuba.

Universidad de Ciencias Médicas de Granma. Facultad de Ciencias Médicas de Manzanillo Celia Sánchez Manduley. Granma. Cuba.
} 
La Organización Mundial de la Salud (OMS) confirma que la prevalencia mundial de la anemia en la población general es del $24,8 \%$, mientras que en los niños en edad preescolar es del 47,4 \%. En Estados Unidos de América, el $9 \%$ de los niños de 12-36 meses presenta déficit de hierro, y el $30 \%$ de estos niños desarrolla una anemia ferropénica. Sin embargo, en regiones con bajos recursos una proporción considerable de niños menores de 5 años padecen esta enfermedad. Reportándose la máxima prevalencia en África $(67,6 \%$ ) y Asia Sudoriental $(65,5 \%)^{3,4}$

Sus causas pueden ser multifactoriales y frecuentemente pueden coexistir varias de ellas; la principal es la baja ingestión de alimentos con fuentes adecuadas de hierro, tanto en cantidad como en calidad. En aquellos menores de un año el destete precoz puede desencadenar la misma, debido a que los lactantes alimentados mediante lactancia materna poseen la ventaja de absorber el hierro con una eficacia 2 o 3 veces superior a los lactantes alimentados con leche de vaca, siendo una de las tantas ventajas que aporta esta práctica ${ }^{3,5,6}$.

En Cuba se trabaja desde el año 1987 en el desarrollo de programas de intervención para la prevención de la anemia por deficiencia de hierro en la población, y se ha dedicado especial atención al Proyecto de Anemia en infantes hasta la edad de cinco años, sin embargo, la anemia por déficit de hierro continúa siendo un problema de salud grave en niños, con nefastas repercusiones en los lactantes menores de un $a \tilde{0} 0^{6}$.

Existe evidencia a nivel mundial de que las intervenciones realizadas para el control de la anemia han logrado evitar sus complicaciones sobre el desarrollo físico y mental de los niños; sin embargo, no se han obtenido los impactos esperados, ya que existen diversos factores que pueden estar incidiendo en ello y a su vez pueden sinergizarse, lo que ha traído como resultado que en casi todos los países en vías de desarrollo siga existiendo un porcentaje elevado de lactantes con anemia, tal como lo señala el Fondo de las Naciones Unidas para la Infancia (UNICEF 2017) $)^{6,7}$.

Esta investigación se desarrolló con el objetivo de caracterizar a los niños menores de un año con anemia ferropénica pertenecientes al Policlínico Docente "7 de Noviembre" del municipio Majibacoa durante el año 2020.

\section{MATERIALES Y MÉTODOS}

Se realizó un estudio observacional, descriptivo, de corte transversal en un universo de niños menores de un año con anemia ferropénica pertenecientes al Policlínico Docente "7 de Noviembre" del municipio
Majibacoa durante el año 2020.

El universo estuvo constituido 42 niños menores de un año con anemia ferropénica pertenecientes al Policlínico Docente "7 de Noviembre", que sus padres mostraron disposición para participar en el estudio. Se trabajó con todo el universo.

Se confeccionó un modelo para la recolección de los datos, el cual fue llenado en cada caso a partir de la información obtenida de las historias clínicas individuales de infantes. Se consideró para el diagnóstico los niños menores de un año con resultado de hemoglobina menor de $11 \mathrm{gr} / \mathrm{dL}$ y presencia de microcitosis, hipocromía y conteo bajo de reticulocitos.

Fueron estudiadas las variables: edad (menores de 6 meses, 7-9, 10-12 meses) y sexo (masculino y femenino), intensidad de la anemia: leve moderada y severa; (leve: Hb entre 10 - 10,9 gr/dL, moderada: 7 - 9,9 gr/dL y severa: $<7 \mathrm{gr} / \mathrm{dL})$ ), factores de riesgo biológicos: desnutrición, enfermedades infecciosas, antecedente de anemia gestacional y bajo peso al nacer, factores de riesgo sociales y culturales: abandono de la lactancia materna exclusiva, ablactación incorrecta, no incorporación de suplementos nutricionales, madres adolescentes, per cápita familiar bajo (se consideró per cápita familiar bajo cuando al sumar todos los ingresos económicos en la familia y dividirlos entre la cantidad de integrantes, este fuera inferior a 500 pesos) y nivel educacional bajo.

El procesamiento y análisis de la información se realizó a través del procesador estadístico SPSS versión 21.0. Para evaluar las variables estudiadas se utilizaron análisis estadísticos descriptivos, tales como frecuencias absolutas y relativas porcentuales.

Esta investigación fue aprobada por el Comité de Ética Médica y el Consejo Científico del Policlínico Docente "7 de Noviembre". Se obtuvo el consentimiento informado de los padres. Durante su realización no se efectuó ninguna intervención terapéutica y se respetó la confidencialidad de los datos obtenidos. Se mantuvo como premisa respetar los principios bioéticos de los estudios con seres humanos, establecidos en la II Declaración de Helsinki y en las normas éticas cubanas.

\section{RESULTADOS}

El $61,9 \%$ de los niños menores de un año con anemia ferropénica eran del sexo femenino; por su parte la edad más representada fue la menor de 6 meses en ambos sexos, aunque más observada en las féminas $(33,3 \%)$. (Tabla 1$)$. 
Tabla 1. Distribución según edad y sexo de los niños menores de un año con anemia ferropénica pertenecientes al Policlínico Docente "7 de Noviembre" del municipio Majibacoa durante el año 2020.

\begin{tabular}{ccccccc}
\multirow{2}{*}{$\begin{array}{c}\text { Edad } \\
\text { (meses) }\end{array}$} & \multicolumn{2}{c}{ Masculino } & \multicolumn{2}{c}{ Femenino } & \multicolumn{2}{c}{ Total } \\
\cline { 2 - 7 } & No. & $\%$ & No. & $\%$ & No. & $\%$ \\
\hline Menores de 6 & 8 & 19,0 & 14 & 33,3 & 22 & 52,4 \\
$7-9$ & 3 & 7,1 & 9 & 21,4 & 12 & 28,6 \\
$10-12$ & 5 & 11,9 & 3 & 7,1 & 8 & 19,0 \\
\hline Total & $\mathbf{1 6}$ & $\mathbf{3 8 , 1}$ & $\mathbf{2 6}$ & $\mathbf{6 1 , 9}$ & $\mathbf{4 2}$ & $\mathbf{1 0 0}$ \\
\hline
\end{tabular}

Fuente: Historias clínicas individuales

El 61,9\% de los niños menores de un año con anemia ferropénica tenían anemia ligera. (Figura 1).

Figura 1. Distribución de los niños menores de un año según la intensidad de la anemia.

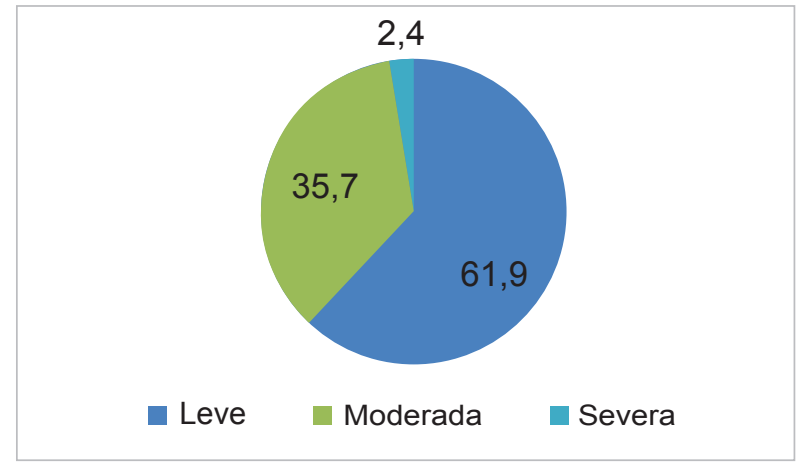

Fuente: Historias clínicas individuales

El 69,0\% de las madres con anemia gestacional sus hijos presentaron anemia ferropénica. (Figura 1).

Figura 2. Distribución de los niños menores de un año con anemia ferropénica según factores de riesgo biológicos.

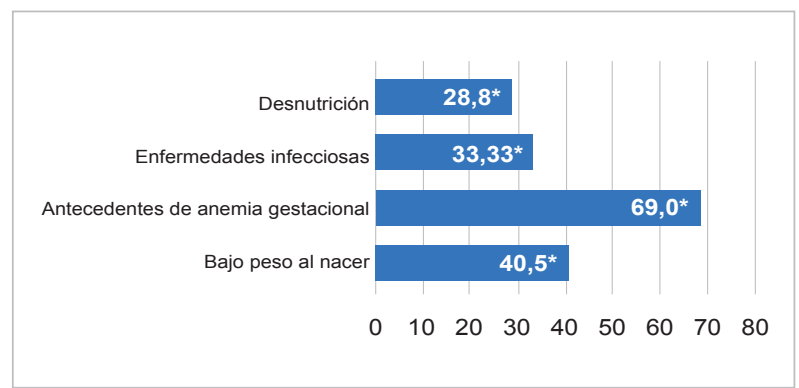

*Porcentaje calculado en relación al total de niños menores de un año con anemia ferropénica

Fuente: historias clínicas individuales

El 47,6\% de los niños menores de un año con anemia ferropénica tenían como antecedente el abandono de la lactancia materna exclusiva. (Tabla 2).
Tabla 2. Distribución de los niños menores de un año con anemia ferropénica según factores de riesgos sociales y culturales.

\begin{tabular}{lcc}
\hline Factores de riesgo sociales y culturales & No. & $\%$ \\
\hline Abandono de la lactancia materna exclusiva & 20 & 47,6 \\
\hline Ablactación incorrecta & 14 & 33,3 \\
\hline No incorporación de suplementos nutricionales & 9 & 21,4 \\
\hline Madres adolescentes & 11 & 29,2 \\
\hline Per cápita familiar bajo & 7 & 16,7 \\
\hline Nivel educacional bajo & 14 & 33,3 \\
\hline
\end{tabular}

*Porcentaje calculado en relación al total de niños menores de un año con anemia ferropénica

Fuente: historias clínicas individuales

\section{DISCUSIÓN}

Los países en vías de desarrollo son los responsables en gran medida de aproximadamente la mitad de las anemias por déficit de hierro, esto hace necesario el reconocimiento de grupos vulnerables y la adopción de medidas paliativas para su prevención y control. Los pacientes con mayor riesgo de padecer anemia carencial en pediatría y en particular ferropénica, son los lactantes, los preescolares y adolescentes; debido al rápido crecimiento que caracterizan estas etapas de la vida y las altas demandas de hierro que de esto se deriva ${ }^{2}$.

Referente al sexo, en el presente estudio se evidenció un predominio del femenino. Resultado similar al descrito por Santamarina A y $\mathrm{col}^{8}$ quienes describieron la enfermedad en el $57,15 \%$ de las féminas. También Molina $\mathrm{N}$ y $\mathrm{col}^{9}$ observaron un mayor porcentaje de pacientes femeninas (55,6 \%); respecto a la edad estos autores preponderaron que, a mayor edad más probabilidad de anemia ferropénica y menor era el aporte de hierro (chi ${ }^{2} 12,2[p=0,03]$ ). Sin embargo, en esta investigación no se comportó como tal, reportándose la mayor cantidad de casos en aquellos menores de 6 meses de edad.

En el presente estudio el mayor porcentaje de niños menores de un año con diagnóstico de anemia ferropénica en cuanto a su intensidad presentaron anemia ligera, resultado similar al encontrado por Iparraguirre-Aliaga $\mathrm{H}^{10}$ quien reporta en el 67,3\% este diagnóstico.

En contraposición, un estudio realizado en Perú ${ }^{11}$ mostró, que el 98,9 \% (558) de los pacientes estudiados presentaron un nivel normal de hemoglobina, frente a una mínima proporción [0,5\% (3)] con anemia leve y moderada, respectivamente. Por su parte, Cruz E y 
$\mathrm{col}^{3}$ identificaron en su estudio la presencia de anemia ferropénica en el $82,6 \%$ de los niños menores de un año; de los cuales fue ligera en el 32,6 \% y moderada en el 50,0 \%. Resultados que distan de la encontrada en este estudio, lo cual puede estar influenciado por la diferencia en los universos de estudios.

Por otra parte, los resultados expuestos no se corresponden con los esfuerzos que realiza Cuba para la prevención de la anemia por déficit de hierro, en el cual se trabaja desde el año 1987 en el desarrollo de programas de intervención para su prevención y se ha dedicado especial atención al "Proyecto de Anemia en infantes hasta la edad de cinco años" 7 .

El abandono de la lactancia materna de forma exclusiva no es recomendable pues la leche materna posee entre 0,3 y $1 \mathrm{mg}$ de hierro por litro y una biodisponibilidad elevada de $50 \%$. Lo que evita en gran mediad la aparición de anemia ferropénica y otras enfermedades frecuentes en estas edades ${ }^{5}$.

Un estudio realizado en Argentina ${ }^{9}$ mostró que el $81 \%$ recibía lactancia materna y solo el $4,5 \%$ de los niños recibían leche no fortificada. Resultados que distan de los obtenidos en este estudio. En similitud, Díaz JA y $\mathrm{col}^{2}$ describen en el 71,3\% de estos niños el abandono de la lactancia materna exclusiva durante el primer semestre, y $68,3 \%$ no se suplementaron con sales ferrosas; haciendo alusión a estos factores como desencadenantes de la anemia ferropénica en los infantes.

En la actualidad se conoce que la anemia materna es un factor de riesgo para el desarrollo de anemia en el lactante de corta edad; sobre todo, si se asocia con otras comorbilidades como el tabaquismo o la diabetes mellitus. Por su parte los productos de gestaciones con anemia en segundo y tercer trimestre tienen elevada probabilidad de padecer este trastorno hemático al nacimiento e incrementarlo durante la vida postnatal, sobre todo, aquellos que no son suplementados oportunamente o provienen de madres con desnutrición no corregida².

Un estudio realizado en Uruguay ${ }^{12}$ expuso que solo el $8,2 \%$ de los menores de un año con anemia ferropénica, sus madres también presentaron anemia durante la gestación, no encontrándose significación estadística [OR=1,13 (0,47-2,77)]. También en Uruguay Assandri $\mathrm{E}$ y col ${ }^{13}$ reportaron en el 32,4 \% antecedente de anemia gestacional; estos autores refieren que este hecho puede constituir ya desde la gestación un factor de riesgo importante para el desarrollo de anemia ferropénica posteriormente en el niño. Las cifras obtenidas en su estudio son inferiores a las descritas en el presente, donde se reportó un elevado número de niños con anemia con antecedentes de madres con anemia gestacional.

Sin embargo, un estudio realizado por Diaz JA y col$^{2}$ reveló que 68 niños $(67,3 \%)$ eran hijos de madres con anemia durante el embarazo. Por otra parte, Santamarina A y $\mathrm{col}^{8}$ plantearon que en su estudio ningún lactante fue bajo peso, ni desnutrido, ni prematuro, ni presentó infecciones a repetición, y todos mantenían la lactancia materna exclusiva, encontrándose como único factor de riesgo a la madre con antecedentes de anemia durante el embarazo (generalmente ligera).

Evidencias científicas nacionales muestran que las insuficiencias en la lactancia materna, el bajo consumo de frutas y vegetales y la deficiencia vitamínica ejercen influencia en la prevalencia de la anemia en Cuba. Además de la ingesta inadecuada de hierro y la baja biodisponibilidad de hierro consumido, como causa fundamental de anemia; la existencia de ciertos tipos de parasitismo intestinal también constituye un factor predisponente al déficit de este micronutriente, lo cual es común a estas edades ${ }^{7}$.

Cóndor-Cisneros $\mathrm{J}$ y col en Perú ${ }^{14}$ realizaron un análisis bivariado sobre los factores de riesgo para el desarrollo de anemia en niños, quienes describen en cuanto a la no lactancia materna exclusiva $[\mathrm{OR}=5,77$ $(2,22-15,02) p=0,0002]$, la inadecuada suplementación con multimicronutrientes [OR= 15,93 $(5,35-47,38)$ $p=0,000]$, y la presencia de enfermedad diarreica aguda $[O R=33,25(9,93-111,28) p=0,000]$, significancia estadística.

En la presente investigación también se identificó como desencadenante de la anemia por déficit de hierro factores relacionados con las madres, como la adolescencia y el bajo nivel escolar; con respecto a esto Molina $\mathrm{N}$ y $\mathrm{col}^{9}$ hacen referencia a la influencia del factor educativo, pues en su estudio mostraron una fuerte asociación entre este factor y desarrollo de la anemia $\left(c^{2}{ }^{2} 7,9[p=0,04]\right)$. No obstante, FernándezOliva $\mathrm{J}$ y col en Perú ${ }^{15}$ observaron que a mayor nivel educativo y edad de la madre se tiene mayor prevalencia de anemia.

Los autores recomiendan la importancia de fomentar en las madres el uso exclusivo durante los primeros seis meses de vida la lactancia materna y de forma complementaria hasta los dos años, además sobre importancia de una dieta saludable y fortificada rica en hierro y otros minerales. Además, realizar actividades de educación nutricional que le brinden a las madres y a la familia en general herramientas relacionadas con la prevención de la anemia ferropénica.

En conclusión, el antecedente de anemia durante la gestación, el bajo peso al nacer, la ablactación 
incorrecta y el abandono de la lactancia materna exclusiva constituyen los principales factores de riesgo en el origen de la anemia ferropénica en niños menores de un año.

Declaración de conflictos de intereses: Los autores declaran no tener conflicto de intereses.

Financiamiento: Autofinanciado.

\section{REFERENCIAS BIBLIOGRÁFICAS}

1. Román Collazo CA, Pardo Vicuña ML, Cornejo Bravo JC, Andrade Campoverde D. Prevalencia de anemia en niños del proyecto EquiDar de la región de Azuay-Ecuador. Rev Cubana de Ped [Internet]. 2018 [citado: 16/7/2021]; 90(1):e360. Disponible en: http://www.revpediatria.sld.cu/ index.php/ped/article/view/706

2. Díaz Colina JA, García Mendiola JJ, Díaz Colina M. Factores de riesgo asociados a la anemia ferropénica en niños menores de dos años. Medimay [Internet]. 2020 [citado: 16/7/2021]; 27(4): 521-530. Disponible en: http://revcmhabana.sld.cu/index.php/rcmh/article/view/1838

3. Cruz Peña E, Arribas Pérez C, Pérez Buchillón M. Factores asociados a la anemia ferropénica en lactantes pertenecientes al Policlínico Concepción Agramonte Bossa. Rev Prog [Internet]. 2019 [citado: 16/7/2021]; 2(3):175-189. Disponible en: http://www.revprogaleno.sld.cu/index.php/ progaleno/article/view/131

4. Fernández-Gonzáles $P$, Hierrezuelo-Rojas N, Monje-Labrada A, Carbó-Cisnero Y. Anemia ferropénica en niños de hasta cinco años de edad atendidos en el policlínico "Ramón López Peña". Rev Elect Dr. Zoilo E. Marinello Vidaurreta [Internet]. 2021 [citado: 16/7/2021]; 46(2). Disponible en: http://revzoilomarinello.sld.cu/index.php/zmv/article/ view/2693.

5. Góngora-Ávila CR, Mejias-Arencibia RA, Vázquez-Carvajal L, Frías-Pérez AE, Cruz-Pérez JL, Cruz-Morales RC. Efectividad de una intervención educativa sobre el nivel de conocimiento de lactancia materna en gestantes. 16 de Abril [Internet]. 2021 [citado: 16/7/2021]; 60 (280): e1206. Disponible en: http://www.rev16deabril.sld.cu/index.php/16_4/ article/view/1244

6. Laborí Quesada P, Laborí Gallego A, Velázquez Reyes M. Caracterización de pacientes en edad pediátrica con anemia ferropénica. Rev Elect Dr. Zoilo E. Marinello Vidaurreta [Internet]. 2017 [citado: 16/7/2021]; 42(3). Disponible en: http://revzoilomarinello.sld.cu/index.php/zmv /article/ view/1076.

7. Pérez-Ávila YF, Pérez-González OF, Yabor-Palomo AM, Laborí-Quesada P, Benítez-Rojas L. Estado nutricional y niveles de hemoglobina en niños menores de cinco años en el área de salud del policlínico "Gustavo Aldereguía Lima". Rev Elect Dr. Zoilo E. Marinello Vidaurreta [Internet]. 2019 [citado: 16/7/2021]; 44(4). Disponible en: http://revzoilomarinello.sld.cu/index.php/zmv/article/view/1870.

8. Santamarina Fernández A, Sánchez Díaz RD, Alba Verdecia O. Caracterización de lactantes menores de 6 meses con anemia ferropénica. Rev Cubana de Ped [Internet]. 2017 [citado: 16/7/2021];89(1):11-19. Disponible en: http:// www.revpediatria.sld.cu/index.php/ped/article/view/168

9. Molina Faveroa N, Rens $V$. Anemia y déficit de hierro en lactantes de 6 a 12 meses de la ciudad de Necochea: prevalencia y determinantes. Arch Argent Pediatr [Internet].
2020 [citado: 16/7/2021]; 118(3):187-192. Disponible en: https://www.sap.org.ar/docs/publicaciones/archivosarg/2020/ v118n3a08.pdf

10. Iparraguirre-Aliaga Hugo. Conocimientos y prácticas de madres sobre prevención de anemia ferropenica en niños menores de 5 años. Hospital Regional de Ica-2019. Rev méd panacea [Internet]. 2020 [citado: 16/7/2021]; 9(2): 105109. Disponible en: https://doi.org/10.35563/r mp.v9i2.328

11. Buitron Martel EL. Estado nutricional y nivel de hemoglobina según el tipo de lactancia en el lactante de 6 meses. Rev Peru Cienc Salud [Internet]. 2021 [citado: 16/7/2021]; 3(1): 13-9. Disponible en: https://doi.org/10.37711/ rpcs.2021.3.1.241

12. Machado K, Alcarraz G, Morinico E, Briozzo T, Gutiérrez S. Anemia ferropénica en niños menores de un año usuarios de CASMU-IAMPP: prevalencia y factores de riesgo. Arch Peditr Urug [Internet]. 2017 [citado: 16/7/2021]; 88(5):254260. Disponibles en: http://www.scielo.edu.uy/scielo.php?script=sci_arttext\&pid=S1688-12492017000500254\&lng=es.

13. Assandri E, Skapino E, Da Rosa D, Alemán A, Acuña AM. Anemia, estado nutricional y parasitosis intestinal en niños pertenecientes a hogares vulnerables de Montevideo. Arch Peditr Urug [Internet]. 2018 [citado: 16/7/2021]; 89(2):8698. Disponible en: https://www.sup.org.uy/archivos-de-pediatria/adp89-2/web/pdf/adp.2018.89.2.a03.pdf

14. Cóndor-Cisneros J, Baldeón-Wong E. Anemia en niños de 6 a 36 meses en un Centro de Salud urbano. Huánuco, 2016. Rev Peru Investig Salud [Internet]. 2019 [citado: 16/7/2021]; 3(3):109-115. Disponible en: http://revistas.unheval.edu.pe/ index.php/repis/article/view/332

15. Fernández-Oliva J, Mamani-Urrutia V. Niveles de hemoglobina en lactantes de 0 a 6 meses de edad hospitalizados en el Instituto Nacional de Salud del Niño, 2015. An Fac med [Internet]. 2019 [citado: 16/7/2021];80(1):45-50. Disponible en: https://doi.org/10.15381/anales. v80i1.15474

\section{Correspondencia:}

César Ramón Góngora-Ávila

Dirección: Calle No. 21 Calixto, Majibacoa Las Tunas.

Cuba

Correo: cesargongora1998@gmail.com

Teléfono: +5351048025 